\title{
Is Resting State Functional MRI Effective Connectivity in Movement Disorders Helpful? A Focused Review Across Lifespan and Disease
}

Running Head: Movement Disorder rs-fMRI Effective Connectivity

Bethany L. Sussman, PhD ${ }^{1}$, Sarah N. Wyckoff, $\mathrm{PhD}^{1,2}$, Jennifer Heim, MD³, Angus A. Wilfong, $\mathrm{MD}^{3}$, P. David Adelson, $\mathrm{MD}^{4}$, Michael C. Kruer, $\mathrm{MD}^{3,5}$, Maria Jose Gonzalez, MD ${ }^{6}$, Varina L. Boerwinkle, $\mathrm{MD}^{3}$

Affiliations:

1. Division of Neurosciences, Barrow Neurological Institute at Phoenix Children's Hospital, 1919 E. Thomas Rd, Phoenix, AZ, 85016 USA

2. Department of Research, Phoenix Children's Hospital, 1919 E. Thomas Rd, Phoenix, AZ 85016 USA

3. Division of Pediatric Neurology, Barrow Neurological Institute at Phoenix Children's Hospital, 1919 E. Thomas Rd, Ambulatory Building, Phoenix, AZ 85016 USA

4. Division of Pediatric Neurosurgery, Barrow Neurological Institute at Phoenix Children's Hospital, 1919 E. Thomas Rd, Phoenix, AZ 85016 USA

5. Departments of Child Health, Neurology, Genetics and Cellular \& Molecular Medicine, University of Arizona College of Medicine - Phoenix, Phoenix, AZ, 85004 USA

6. Departments of Pediatrics, SBH Health System, Bronx, NY, 10457 USA

Corresponding author:

Maria Jose Gonzalez, MD

Barrow Neurological Institute at Phoenix Children's Hospital

1919 E. Thomas Rd. Ambulatory Building

Phoenix, AZ 85016

602-933-2115(phone)

602-933-4253 (fax)

mariajose.gonzalezvill@gmail.com

Keywords: resting state functional MRI, effective connectivity, dystonia, movement disorders, subcortical, basal ganglia. 


\section{Abstract}

In the evolving modern era of neuromodulation for movement disorders in adults and children, much progress has been made recently characterizing the human motor network (MN) with potentially important treatment implications. Herein is a focused review of relevant resting state fMRI functional and effective connectivity of the human motor network across the lifespan in health and disease. The goal is to examine how the transition from static functional to dynamic effective connectivity may be especially informative of network-targeted movement disorder therapies, with hopeful implications for children.

\section{Impact Statement}

While functional connectivity has elucidated much MN properties with relation to age, disease, and behavior, effective connectivity has been shown to be useful in $\mathrm{MN}$-informed therapies in adults. Thus, effective connectivity may have potential to impact childhood movement disorder therapies, given the lower to no patient demand. 


\section{Functional Connectivity}

\section{Healthy adults}

Studies of motor network (MN) resting state fMRI (rs-fMRI) functional connectivity (FC) build upon those from structural connectivity (SC) in healthy adults and together show that flexible motor behavior is generated and tightly regulated by sophisticated physiological networks residing in the cortex and subcortical structures through a series of parallel loops, known as the direct, indirect, and hyperdirect pathways. ${ }^{1-9}$ Although these network loops are configured in parallel, as evidenced by neuron tracing studies in primates and non-primate animal models and corroborated in diffusion tensor imaging (DTI) tractography in healthy adults, they are not independent. ${ }^{4-6,10}$ Such parallel yet interdependent networks yield means to compensate for local network pathology. Evidence for the compensation-yielding loops is shown through isolated lesions of pallidum and striatum in adults, which are not always associated with severe motor dysfunction. ${ }^{10,11}$ Thus, the static measures of SC and FC have provided additional insight and support of prior modalities' findings of MN configuration and function.

\section{Age dependency}

FC has revealed age-dependent basal ganglia $(B G)$ and related motor phenotype differences. BG FC primarily increases from age seven to middle adulthood and then decreases in the healthy aging elderly. $2,12-14$ However, in one study in the healthy elderly no relationship between BG FC and motor skill was detected. ${ }^{14}$ Changes in FC from early to mid-adulthood are more varied between the BG and cortical regions than between subcortical motor regions, indicating the subcortical and cortical motor structures have different age-dependent connectivity trajectories in adulthood. ${ }^{2}$ Thus, static FC across development does have age-related changes that correlate with motor behavior, though may have no significant behavioral relationship in the age group of older healthy adults. 


\section{Behavior}

In the healthy, MN FC is associated with motor skill and training. A recent study of trained young adult dancers showed that, compared to non-dancers, dancers had higher FC in bilateral sensorimotor cortex, bilateral putamen, and right superior occipital gyrus. ${ }^{15}$ They also had increased FC between the putamen and middle cingulate cortex (MCC), and enhanced integration of cortico-basal ganglia loops via the MCC. Additionally, FC in the sensorimotor cortex was positively correlated with the average time spent training in a week. This suggests that FC in these regions is related to the amount of time spent using motor programs and regions, which is relevant for clinical conditions that may affect those regions. A study investigating FC in musicians with and without musician's dystonia found that, FC within in the right putamen was increased overall in musicians with musician's dystonia compared to musicians without musician's dystonia (hyperconnectivity), and that this difference was greatest in the right anterior putamen. However, increased FC within the anterior right putamen was correlated with piano skill playing level in healthy controls $(\mathrm{HC})$, but not those with musician's dystonia. ${ }^{16}$ Thus, static MN FC is associated with and is modulated by MN-utilization behaviors, and this appears to be distinguishable from disease-related FC.

\section{Disease}

MN FC demonstrates disease differentiating and severity attributes. Parkinson's disease showed decreased BG FC (hypoconnectivity), compared to HC and Alzheimer's disease. Thus, Alzheimer's and Parkinson's disease are differentiable by FC. ${ }^{17}$ Parkinson's Disease shows reduced FC between striatum and the thalamus, midbrain, pons, and cerebellum have also been noted in PD compared to HC, whereas Alzheimer's disease has less specifically localized neurodegenerative FC effect. ${ }^{17,18}$ Parkinson's disease FC has shown additional broader range reduced connectivity in the supplementary motor area (SMA), left dorsolateral prefrontal cortex 
(DLPFC), and left putamen, and increased FC in the left primary motor cortex (M1), parietal cortex, and cerebellum compared to HC..$^{19}$ In Parkinson's disease midbrain-cortical FC is associated with freezing gait. ${ }^{20}$ Neonatal prematurity showed association between thalamusmotor and BG-motor network connectivity and behavioral motor outcome scores in a systemic review. ${ }^{21}$ Neonatal diaschisis resulting in later outcomes of severe childhood motor impairments are associated with MN FC. ${ }^{22}$ Thus, static MN FC in neonatal, pediatric, and adult pathology is associated with motor impairments and adult MN pathologies of Parkinson's and Alzheimer's disease show FC-disease differentiating attributes and widespread network effects.

Static FC has also demonstrated differences between patients with focal dystonia and HC and that these differences can be specific to the type of focal dystonia. Right-handed patients with writers' cramp show FC decreases in the left primary somatosensory area as well as between the left superior parietal lobule and left dorsal premotor hand area and FC increases in left putamen Mohammadi et al. (2012). ${ }^{23,24}$ In contrast, patients with spasmodic dysphonia have been shown to have a mixture of increased and decreased FC between deep grey, cerebellar, and cortical regions thought to subserve language and articulation compared to controls and FC between left thalamus and left caudate was correlated with disease severity. ${ }^{25}$ In cervical dystonia, patients were shown to have decreased FC between the primary and secondary sensory cortices compared to controls, but increased FC within the BG, between the $B G$ and thalamus, and between the somatosensory and motor cortices. ${ }^{26}$ In contrast, patients with blepharospasm showed only widespread reduced FC, both regional (between neighboring cortical or cerebellar regions, but not within deep grey structures) and long-range. ${ }^{27}$ Thus, FC abnormalities in focal dystonia appear to show some differentiation between focal dystonia type.

There is also evidence that abnormal regional FC contributes to disruption of global FC.$^{28}$ However, another study determined that while patients with focal dystonia had decreased functional connectivity regionally (within the striatum and between lateral primary sensorimotor 
cortex and the ventral intraparietal area), whole-brain global FC measures did not distinguish dystonia from $\mathrm{HC}$ after motion correction using global signal regression. ${ }^{29}$ In summary, focal dystonia in adults also show FC differences compared to controls, these differences appear to be primarily hyper connective within deep gray structures and often, but not always, hypoconnective in the cerebral cortex.

There have been several recent advances in knowledge about the potential utility of FC in predicting the effectiveness of deep brain stimulation (DBS) in Parkinson's disease, in particular, including the development of specialized toolboxes that provide pipelines for processing an integrating neuroimaging data for DBS. ${ }^{30,31}$ Using a combination of normative healthy adult data and data from adults with Parkinson's disease, Horn et al. (2017) demonstrated that preoperative anti-correlated FC between the subthalamic nucleus (DBS lead target) and primary motor cortex is predictive of clinical improvement after STN-DBS implantation. ${ }^{32}$ Preoperative FC between STN and the ipsilateral globus pallidus internus (GPi) has also been shown to be positively correlated with clinical improvement after STN-DBS in Parkinson's disease. ${ }^{33}$ In contrast, there is far less known about preoperative FC and DBS outcomes in patients with idiopathic generalized dystonia, however a recent study positively correlated preoperative FC between lead site (GPi) and primary sensorimotor cortex, motor thalamus, and cerebellum. ${ }^{34}$ It should be noted that several of these connectivity studies also utilize structural connectivity (T2 diffusion imaging) although FC often independently predicts DBS outcomes. ${ }^{32,34}$

Finally, studies have also shown changes in FC to be associated with treatment for movement disorders. While this proves challenging for DBS (a pre-post FC design is technically difficult due to the challenges of MRI after DBS including both safety and susceptibility artifact introduced from DBS lead sites), there have been some observational studies investigating FC changes after treatment with botulinum toxin in focal (cervical) dystonia and 
blepharospasm. ${ }^{26,27,35-37}$ Taken together, these studies demonstrate that brain network changes associated with clinical treatment in movement disorders can be respectively measured with FC.

\section{Advancements through Effective Connectivity}

Static FC demonstrates the potential of rs-fMRI signal to capture MN relevant characterizations. Although FC has revealed MN location and connectivity changes with age and disease, a different rs-fMRI connectivity approach, EC, may be uniquely informative for clinically relevant dynamic $\mathrm{MN}$-informed characterizations. Importantly, EC allows the user to not only infer functional connectivity between two regions, but direction of influence. This may prove beneficial for understanding not only why regions appear hyper or hypoconnected but also the mechanistic changes of treatments.

An important aside on EC methods: active task-based EC (active-EC) requires patient cooperation, whereas passive task-based EC (passive-EC) and purely resting state EC (rs-EC) have little to no patient cooperation demand, respectively, which has age-capacity success implications.

\section{Healthy adults}

Supportively, healthy adults' active-EC is predictive of immediate future motor performance and downstream signaling network paradigms. For example, during adult response inhibition active-EC increases from the globus pallidus internus to the thalamus, predicting both increased response inhibition and downstream active-EC from pre-SMA to right caudate. ${ }^{38}$ Such immediate-behavioral-and-MN prediction capacity could hypothetically inform adjustments deep brain stimulator (DBS) neuromodulation settings in disease. Also, this dynamic active-EC signaling paradigm is consistent with the indirect and hyperdirect model of BG signaling inhibition. ${ }^{38}$ These active-EC measures also correlated with the Unified Parkinson's Disease Rating Scale motor score and the finding that use of levodopa normalizes atypical FC patterns. ${ }^{38}$ 
Taken together, this suggests that active-EC measures have pathophysiologic meaning and could hypothetically accelerate DBS motor related improvements via informed stimulation parameter adjustments.

\section{Disease}

Additionally, altered active-EC and passive-EC in adult BG circuitry has been observed in movement disorders including PD (passive-EC, and active-EC), and dystonia (active-EC). ${ }^{39-43}$ For example, adults with writer's cramp dystonia compared to HC showed a mixture of abnormal excitatory and inhibitory active-EC within connections between motor cortex and the cerebellum, as well as BG during a finger tapping task with the non-dominant hand. ${ }^{43}$ Thus, active-EC was able to localize the atypical connections during task. Additionally, in adults with spasmodic dysphonia, both top-down (parieto-putaminal) and interhemispheric (right-to-left pre-motor) rs-EC is hyperexcitable and patients with and without tremor can be further differentiated by the fact that patients with tremor additionally show less self-inhibition for the left parietal cortex, left putamen, and right premotor cortex. ${ }^{44}$ Thus rs-EC was able to localize atypical directional connectivity in focal dystonia as well as differentiate sub-groups of similar patient groups.

Importantly, passive-EC and rs-EC have demonstrated potential for localizing and characterizing pathological dynamic network signal on an individual basis to guide effective surgical therapies in adults, such as the positioning of modulatory intracerebral leads or ablations. 40,45 Such proof of concept is exemplified in PD, where passive-EC by dynamic causal modeling (DCM) has been used to model the effects of subthalamic nucleus (STN)-DBS on BG circuitry, and changes of directional modulation induced with 'on' STN-DBS are associated with clinical efficacy of DBS. ${ }^{40}$

One advantage of passive and rs-EC paradigms for investigating the effects of DBS is that directional computational modelling techniques, such as dynamic causal modelling (DCM), can 
model 'hidden' nodes in a network. This is beneficial because, post DBS implant, an MRI susceptibility artifact will occur at lead locations, thus making it not possible to measure the BOLD response at lead location. However, as techniques such as DCM are able to model lead-inferred location activity (LILA) within a network of regions by placing a 'hidden' node at the lead location, in can use rs-fMRI to infer the effect of DBS on brain-network dynamics. ${ }^{40,46,47}$ rs-EC in essential tremor, pre and post-thalamotomy, also predicts post-operative dynamic MN changes and clinical motor-symptom scores. ${ }^{45}$ This suggests that rs-EC characterizes casual network dynamics with high enough integrity to guide surgical strategy and is associated with clinical outcomes in adults with essential tremor. Specifically, rs-EC allows for the identification of regions that are functionally connected, as well as the direction and nature of those connections. Certain techniques also allow for LILA, opening possibilities for inferring neural activity at lead sites and identifying causal network dynamic changes (not restricted to lead sites) as a function of DBS. As such, rs-EC may lead to higher specificity and precision in identifying underlying network neuropathology.

\section{Conclusion}

Functional connectivity builds upon the work established by structural modalities in characterizing MN configuration, is associated with lifespan MN related changes, has pathologylocalizing potential, has potential to localize FC related changes after medical treatments including medications and botulinum injection, and relates to motor behavior in health and disease across neonatal to adult age groups. However, effective connectivity may prove to be particularly useful in brain-invasive (DBS) MN-informed therapies due to its potential to demonstrate lead-inferred location activity, especially as DBS systems have begun to be compatible with 3T MRI. PassiveEC and rs-EC potential may hold promise in childhood movement disorder therapies, given the lower to no patient demand during a resting state fMRI scan. 


\section{Authors' Disclosure Statement}

None of the authors have any conflicts to disclose.

\section{Funding Statement}

The authors have no funding to declare. 


\section{References}

1. Barnes KA, Cohen AL, Power JD, Nelson SM, Dosenbach YB, Miezin FM, et al. Identifying basal ganglia divisions in individuals using resting-state functional connectivity MRI. Front Syst Neurosci. Published online 2010. doi:10.3389/fnsys.2010.00018

2. Manza P, Zhang S, Hu S, Chao HH, Leung HC, Li C shan R. The effects of age on resting state functional connectivity of the basal ganglia from young to middle adulthood. Neurolmage. 2015;107:311-322. doi:10.1016/j.neuroimage.2014.12.016

3. Robinson $\mathrm{S}$, Basso $\mathrm{G}$, Soldati $\mathrm{N}$, et al. A resting state network in the motor control circuit of the basal ganglia. BMC Neurosci. 2009;10(1):137. doi:10.1186/1471-2202-10-137

4. Cacciola A, Milardi D, Bertino S, et al. Structural connectivity-based topography of the human globus pallidus: Implications for therapeutic targeting in movement disorders. Mov Disord. 2019;34(7):987-996. doi:10.1002/mds.27712

5. Alexander GE, DeLong MR, Strick PL. Parallel Organization of Functionally Segregated Circuits Linking Basal Ganglia and Cortex. Annu Rev Neurosci. 1986;9(1):357-381. doi:10.1146/annurev.ne.09.030186.002041

6. Haber SN. The primate basal ganglia: parallel and integrative networks. J Chem Neuroanat. 2003;26(4):317-330. doi:10.1016/j.jchemneu.2003.10.003

7. Milardi D, Quartarone A, Bramanti A, et al. The Cortico-Basal Ganglia-Cerebellar Network: Past, Present and Future Perspectives. Front Syst Neurosci. 2019;13:61. doi:10.3389/fnsys.2019.00061

8. Albin RL, Young AB, Penney JB. The functional anatomy of basal ganglia disorders. Trends Neurosci. 1989;12(10):366-375. doi:10.1016/0166-2236(89)90074-X

9. Nambu A, Tokuno H, Takada M. Functional significance of the cortico-subthalamo-pallidal 'hyperdirect' pathway. Neurosci Res. 2002;43(2):111-117. doi:10.1016/S01680102(02)00027-5

10. Quartarone A, Cacciola A, Milardi D, et al. New insights into cortico-basal-cerebellar connectome: clinical and physiological considerations. Brain. Published online October 19, 2019:awz310. doi:10.1093/brain/awz310

11. Bhatia KP, Marsden CD. The behavioural and motor consequences of focal lesions of the basal ganglia in man. Brain. 1994;117(4):859-876. doi:10.1093/brain/117.4.859

12. Allen EA, Erhardt EB, Damaraju E, et al. A Baseline for the Multivariate Comparison of Resting-State Networks. Front Syst Neurosci. 2011;5. doi:10.3389/fnsys.2011.00002

13. Solé-Padullés $C$, Castro-Fornieles J, de la Serna $E$, et al. Intrinsic connectivity networks from childhood to late adolescence: Effects of age and sex. Dev Cogn Neurosci. 2016;17:35-44. doi:10.1016/j.den.2015.11.004 
14. Griffanti L, Stratmann P, Rolinski M, et al. Exploring variability in basal ganglia connectivity with functional MRI in healthy aging. Brain Imaging Behav. 2018;12(6):1822-1827. doi:10.1007/s11682-018-9824-1

15. Li G, He H, Huang M, et al. Identifying enhanced cortico-basal ganglia loops associated with prolonged dance training. Sci Rep. 2015;5(1):10271. doi:10.1038/srep10271

16. Kita K, Rokicki J, Furuya S, Sakamoto T, Hanakawa T. Resting-state basal ganglia network codes a motor musical skill and its disruption From dystonia: Resting-State Network in Musicians. Mov Disord. 2018;33(9):1472-1480. doi:10.1002/mds.27448

17. Rolinski M, Griffanti L, Szewczyk-Krolikowski K, et al. Aberrant functional connectivity within the basal ganglia of patients with Parkinson's disease. Neurolmage Clin. 2015;8:126-132. doi:10.1016/j.nicl.2015.04.003

18. Hacker CD, Perlmutter JS, Criswell SR, Ances BM, Snyder AZ. Resting state functional connectivity of the striatum in Parkinson's disease. Brain. 2012;135(12):3699-3711. doi:10.1093/brain/aws281

19. Wu T, Wang L, Chen Y, Zhao C, Li K, Chan P. Changes of functional connectivity of the motor network in the resting state in Parkinson's disease. Neurosci Lett. 2009;460(1):6-10. doi:10.1016/j.neulet.2009.05.046

20. Droby A, Pelosin E, Putzolu M, et al. A Multimodal Imaging Approach Demonstrates Reduced Midbrain Functional Network Connectivity Is Associated With Freezing of Gait in Parkinson's Disease. Front Neurol. 2021;12:583593. doi:10.3389/fneur.2021.583593

21. Wheelock MD, Austin NC, Bora S, et al. Altered functional network connectivity relates to motor development in children born very preterm. Neurolmage. 2018;183:574-583. doi:10.1016/j.neuroimage.2018.08.051

22. Ishaque $\mathrm{M}$, Manning $\mathrm{JH}$, Woolsey $\mathrm{MD}$, et al. Functional integrity in children with anoxic brain injury from drowning. Hum Brain Mapp. 2017;38(10):4813-4831. doi:10.1002/hbm.23745

23. Mohammadi B, Kollewe K, Samii A, Beckmann CF, Dengler R, Münte TF. Changes in restingstate brain networks in writer's cramp. Hum Brain Mapp. 2012;33(4):840-848. doi:10.1002/hbm.21250

24. Delnooz CCS, Helmich RC, Toni I, van de Warrenburg BPC. Reduced parietal connectivity with a premotor writing area in writer's cramp: Reduced Parietal Coupling in Writer's Cramp. Mov Disord. 2012;27(11):1425-1431. doi:10.1002/mds.25029

25. Kiyuna A, Kise N, Hiratsuka M, et al. Brain Activity in Patients With Adductor Spasmodic Dysphonia Detected by Functional Magnetic Resonance Imaging. $J$ Voice. 2017;31(3):379.e1-379.e11. doi:10.1016/j.jvoice.2016.09.018

26. Brodoehl S, Wagner F, Prell T, Klingner C, Witte OW, Günther A. Cause or effect: Altered brain and network activity in cervical dystonia is partially normalized by botulinum toxin treatment. Neurolmage Clin. 2019;22:101792. doi:10.1016/j.nicl.2019.101792 
27. Jochim A, Li Y, Gora-Stahlberg G, et al. Altered functional connectivity in blepharospasm/orofacial dystonia. Brain Behav. 2018;8(1):e00894. doi:10.1002/brb3.894

28. Battistella G, Termsarasab P, Ramdhani RA, Fuertinger S, Simonyan K. Isolated Focal Dystonia as a Disorder of Large-Scale Functional Networks. Cereb Cortex. Published online December 17, 2015:bhv313. doi:10.1093/cercor/bhv313

29. Norris SA, Morris AE, Campbell MC, et al. Regional, not global, functional connectivity contributes to isolated focal dystonia. Neurology. 2020;95(16):e2246-e2258. doi:10.1212/WNL.0000000000010791

30. Horn A, Kühn AA. Lead-DBS: A toolbox for deep brain stimulation electrode localizations and visualizations. Neurolmage. 2015;107:127-135. doi:10.1016/j.neuroimage.2014.12.002

31. Horn A, Li N, Dembek TA, et al. Lead-DBS v2: Towards a comprehensive pipeline for deep brain stimulation imaging. Neurolmage. 2019;184:293-316. doi:10.1016/j.neuroimage.2018.08.068

32. Horn A, Reich M, Vorwerk J, et al. Connectivity Predicts deep brain stimulation outcome in Parkinson disease: DBS Outcome in PD. Ann Neurol. 2017;82(1):67-78. doi:10.1002/ana.24974

33. Younce JR, Campbell MC, Hershey T, et al. Resting-State Functional Connectivity Predicts STN DBS Clinical Response. Mov Disord. 2021;36(3):662-671. doi:10.1002/mds.28376

34. Okromelidze L, Tsuboi T, Eisinger RS, et al. Functional and Structural Connectivity Patterns Associated with Clinical Outcomes in Deep Brain Stimulation of the Globus Pallidus Internus for Generalized Dystonia. Am J Neuroradiol. 2020;41(3):508-514. doi:10.3174/ajnr.A6429

35. Delnooz CCS, Pasman JW, Beckmann CF, van de Warrenburg BPC. Task-Free Functional $\mathrm{MRI}$ in Cervical Dystonia Reveals Multi-Network Changes That Partially Normalize with Botulinum Toxin. Barton JJS, ed. PLOS ONE. 2013;8(5):e62877. doi:10.1371/journal.pone.0062877

36. Feng L, Yin D, Wang $X$, et al. Brain connectivity abnormalities and treatment-induced restorations in patients with cervical dystonia. Eur $J$ Neurol. 2021;28(5):1537-1547. doi:10.1111/ene. 14695

37. Hok $P$, Hvizdošová $L$, Otruba $P$, et al. Botulinum toxin injection changes resting state cerebellar connectivity in cervical dystonia. Sci Rep. 2021;11(1):8322. doi:10.1038/s41598021-87088-z

38. Jahfari S, Waldorp L, van den Wildenberg WPM, Scholte HS, Ridderinkhof KR, Forstmann BU. Effective Connectivity Reveals Important Roles for Both the Hyperdirect (FrontoSubthalamic) and the Indirect (Fronto-Striatal-Pallidal) Fronto-Basal Ganglia Pathways during Response Inhibition. J Neurosci. 2011;31(18):6891-6899. doi:10.1523/JNEUROSCI.525310.2011

39. Dirkx MF, den Ouden H, Aarts E, et al. The Cerebral Network of Parkinson's Tremor: An Effective Connectivity fMRI Study. J Neurosci. 2016;36(19):5362-5372. doi:10.1523/JNEUROSCI.3634-15.2016 
40. Kahan J, Urner M, Moran R, et al. Resting state functional MRI in Parkinson's disease: the impact of deep brain stimulation on 'effective' connectivity. Brain. 2014;137(4):1130-1144. doi:10.1093/brain/awu027

41. Rowe JB, Hughes LE, Barker RA, Owen AM. Dynamic causal modelling of effective connectivity from fMRI: Are results reproducible and sensitive to Parkinson's disease and its treatment? Neurolmage. 2010;52(3):1015-1026. doi:10.1016/j.neuroimage.2009.12.080

42. Duggan O, Narasimham S, Govern EM, Killian O, O'Riordan S, Hutchinson M, et al. A Study of the Midbrain Network for Covert Attentional Orienting in Cervical Dystonia Patients using Dynamic Causal Modelling. In: ; 2019.

43. Rothkirch I, Granert O, Knutzen A, et al. Dynamic causal modeling revealed dysfunctional effective connectivity in both, the cortico-basal-ganglia and the cerebello-cortical motor network in writers' cramp. Neurolmage Clin. 2018;18:149-159. doi:10.1016/j.nicl.2018.01.015

44. Battistella G, Simonyan K. Top-down alteration of functional connectivity within the sensorimotor network in focal dystonia. Neurology. 2019;92(16):e1843-e1851. doi:10.1212/WNL.0000000000007317

45. Park HJ, Pae C, Friston K, et al. Hierarchical Dynamic Causal Modeling of Resting-State fMRI Reveals Longitudinal Changes in Effective Connectivity in the Motor System after Thalamotomy for Essential Tremor. Front Neurol. 2017;8:346. doi:10.3389/fneur.2017.00346

46. Daunizeau J, Friston KJ, Kiebel SJ. Variational Bayesian identification and prediction of stochastic nonlinear dynamic causal models. Phys Nonlinear Phenom. 2009;238(21):20892118. doi:10.1016/j.physd.2009.08.002

47. Friston KJ, Harrison L, Penny W. Dynamic causal modelling. Neurolmage. 2003;19(4):12731302. doi:10.1016/S1053-8119(03)00202-7 